\title{
Monitoring the Displacement the Roof of Underground Mines for Safety of Mining Operations
}

\author{
Maxim Inozemtsev ${ }^{1, *}$, Svetlana Nikitina $^{1}$, and Mikhail Kononov ${ }^{1}$ \\ ${ }^{1}$ T. F. Gorbachev Kuzbass State Technical University, Prokopievsk branch, 653039 Nogradskaya str. \\ 19a, Prokopievsk, Russia
}

\begin{abstract}
In the article the necessity of continuous control over the condition of the roof of mine workings is considered, to increase the safety in the conduct of mining operations. Provided the rationale for monitoring in complex mining and geological conditions, as well as in areas prone to rock blows and sudden coal emissions. The existing methods for controlling the displacement of the roof rocks are described, and their shortcomings are given. An idea is given of an automated system for monitoring the displacement of the workings. The stages of the system as a whole are considered, including the choice of a linear displacement sensor, a platform for software development, and a programming language. In order to ensure integration into other systems and subsequent analysis of the results, it is envisaged to output data to spreadsheets. Are shown the interfaces of the program and the output of the readings from the sensors to the monitors of the mining manager.
\end{abstract}

\section{Introduction}

Improving the safety of coal production remains an important problem given the growing concentration of equipment and mining operations. One of the factors of possible accidents is to develop areas that are dangerous on the mountain blows and sudden outbursts. The aim of determining the zone of destruction of a rock mass around an excavation and the pillars. The shape and dimensions of fracture zone depends on the prevailing stresses and rock strength, depth of development, presence of geological disturbances.

Sudden geodynamic manifestations in the roof of mine workings by a number of factors: the geological irregularities of the rocks, excessive hydration of the array, the excess load on the anchor bolts with the simultaneous mining of multiple seams and inconsistencies of the lining according to geological conditions, are some of many of these factors. A big step to solving this problem is to identify the causes of the collapse. Sometimes the cause of roof collapse can't be determined, but the study of falling of the detached and loosened rocks, the analysis of the environmental and geotechnical environment can identify the causes of the collapse [1].

\footnotetext{
* Corresponding author: inozemcevma@kuzstu.ru
} 
An increasing amount of coal produced, increasing the depth of excavations require improved methods of monitoring the condition of the roof, with high reliability of results, efficiency and continuity of control.

In the situation of safe mining is regulated by conducting research on each field, inclined or threat on the mountain bumps, to improve prediction and prevention of rockbursts. The forecast rockburst hazard areas of the rock mass should be carried out as according to the system of continuous automated control of the stress state of the array and change the values (speeds) deformations and displacements.

Responsible equipment, such as ventilation systems of coal mines supplied with stationary vibration, temperature and performance sensors [2, 3]. Information from the sensors is incorporated into a single multifunction system security. In this same system it is proposed to make information about the condition of mine workings.

The diversity of geological and geotechnology factors continues to create difficulty in forecasting the manifestations of rock pressure.

To identify regularities of rock pressure, early detection of difficult situations for the management of rock pressure and the lining work, the adoption of measures to prevent accidents of mining operations, it is essential to acquire information and create a database with the resulting parameters, magnitude, and rate of displacement of the roof sections. Efficient work is possible with the use of the system for continuous monitoring of displacements of roof, impact analysis of various factors, and release of information about dangerous sections of the mine workings, where it is necessary to take measures to strengthen the attachment.

\section{Materials \& Methods}

At present, methods of numerical simulation of geomechanical processes are being conducted, cores are selected, the structure of the roof rocks is analyzed using a video endoscope and GPR method [4-6].

In addition, today at the enterprises of extracting coal by underground methods, for monitoring the displacement of rocks in mines used reference deep stations, which allow for visual contact to detect the displacement of the rock mass. Method visual inspection with the help of the deep reference stations is to monitor the rock movement by recording readings in displacement of reference points fixed in the hole of the roof at an appropriate distance [5].

At the moment this method is outdated and has a number of disadvantages [6-9]:

1. For a particular clear information need to continuously monitor the reference points, i.e. directly it is necessary to continuously record the incident displacement.

2. The uncertainty of the exact time of occurrence of the displacement of the rock mass, as well as the lack of information on the speed of its displacement.

Based on this, it becomes clear that engineering employee section, verify the state of the reference deep stations during the shift, the sudden and significant displacement of rocks of the roof may not be able to warn other employees of the mine. As a rule, it makes Overman times per shift, and say how long was the movement, is not possible [6].

The researchers' findings [8-15] show the relevance of the automated monitoring system, with different implementation methods.

Thus, our main task is to create a comprehensive automatic system of monitoring and recording the displacement of the roof of underground mines to improve safety of mining operations, which directly will include:

1. Displacement sensors;

2. Transmitting devices;

3. Communication channels; 


\section{Software;}

Data output to the mine dispatcher monitors.

Direct work on creation of the automated monitoring system will contain the following stages:

1. Replacement of the displacement sensor.

2. The choice of the transmitting device.

3. Definition a method of data transfer (data transfer channels).

4. Definition of platform software development.

5. Software development.

The displacement sensor is a device used to determine the amount of linear or angular mechanical displacement of any object. It should be noted that all of the displacement sensors can be divided into two main categories: sensors linear displacement and angular displacement transducers (encoders). From all existing types of sensors we are more suited to the linear encoders.

After analyzing the advantages and disadvantages of various transducers, relative to the required parameters it can be concluded that the most appropriate as a displacement sensor roof are capacitive displacement transducers with the changing area of the plates. The main advantages of capacitive transducers - ease of use, high sensitivity, low power consumption, absence of moving contacts, comparative ease of fabrication, small size and weight, long service life.

At the stage of approach to software development, we have built a common schema representation of the system as a whole, the diagram (see Fig. 1).

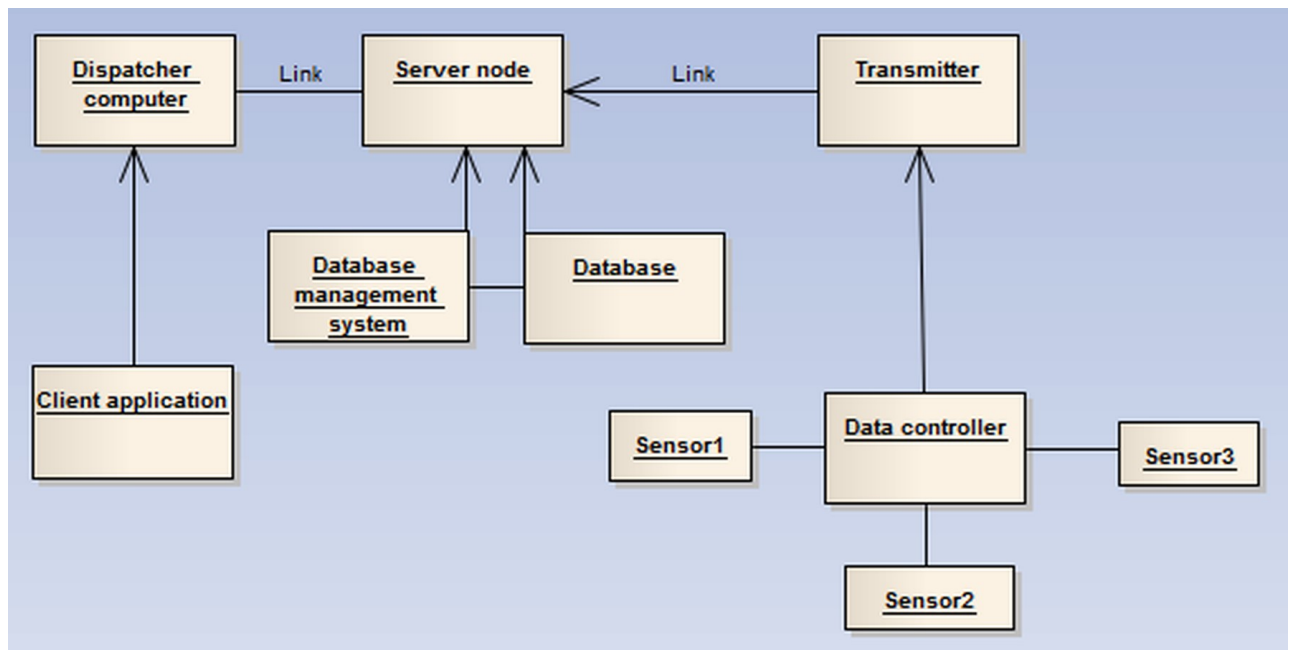

Fig. 1. System representation diagram.

\section{Results and Discussion}

At the software development stage, the subject area was first analyzed. The most important point to which we drew attention is very large volumes of information, and it was decided to store all non-recurring recorded rock mass readings taken from each displacement sensor.

The architecture of the information system being developed is the client-server architecture. This architecture is implemented by the database management system (DBMS) of MSSQL Server.

Using this DBMS will provide the following functions: 
- Ensures the integrity of the database (DB);

- provides fast recovery after various failures (hardware and software);

- provides backup;

- provides high operational reliability;

- provides high performance.

During the development of the database it was decided to create a table for each sensor and thus the following information objects were identified:

- Sensor 1

- Sensor 2

- Sensor $\mathrm{n}$

Integrity requirement: to table contents Sensor 1, Sensor 2, Sensor n must necessarily refer to level 1, level 2, date and time of reading, for two-level sensors. As a result, we got a rational database for storing information, a database diagram (see Fig. 2).

\begin{tabular}{|l|}
\hline Sensor1_Table \\
\hline Level1: double \\
\hline Level2: double \\
\hline Date_And_Time: datetime \\
\hline
\end{tabular}

\begin{tabular}{|l|}
\hline Sensor2_Table \\
\hline Level1: double \\
\hline Level2: double \\
\hline Date_And_Time: datetime \\
\hline
\end{tabular}

\begin{tabular}{|l|}
\hline SensorN_Table \\
\hline Level1: double \\
\hline Level2: double \\
\hline Date_And_Time: datetime \\
\hline
\end{tabular}

Fig. 2. Resulting ER diagram (entity diagram-connection) of the database.

After implementing the data warehouse, we went on to the process of creating the program itself.

As technology of interaction of user-defined components with data, ADO.NET technology is chosen - the main model of data access for applications based on Microsoft .NET. The choice of technology is based on the choice of operating system. As a programming environment, choose Visual Studio, and as the language of $\mathrm{C} \#$. This language and environment are universal programming tools, so they are suitable for solving the set task of creating a system. The development of an implementation model (program code) can be represented as a description of the components that implement the boundary object classes and entity classes.

The implementation model includes the components integrated into the subsystems that implement the objects identified in the design process.

For each form and bookmark on the form, the composition of interactions with the user is determined, thanks to which the process of receiving and accounting of the readings of the sensors is carried out, as well as notification of the controller about the displacement of each sensor in a particular area.

To fulfill each functional requirement, we select according to a separate scenario. Scenario for fulfilling the functional requirement of finding the readings of the selected sensor for the entire time since the introduction of the system and installing the sensor:

The dispatcher has the ability to plot the selected sensor for the entire time, from the moment of the sensor introduction, as well as for the day, week, month, year.

To implement this scenario, two stored procedures were created in the database, which will be called in a program created on the Windows platform. As part of the system being developed, the client component and the server component are distinguished. The server component implements scenarios related to access to the data and its main processing. The client component implements the presentation of data in a user-friendly form and selects the actions to be performed.

The developed interface of the program is presented in Fig. 3 


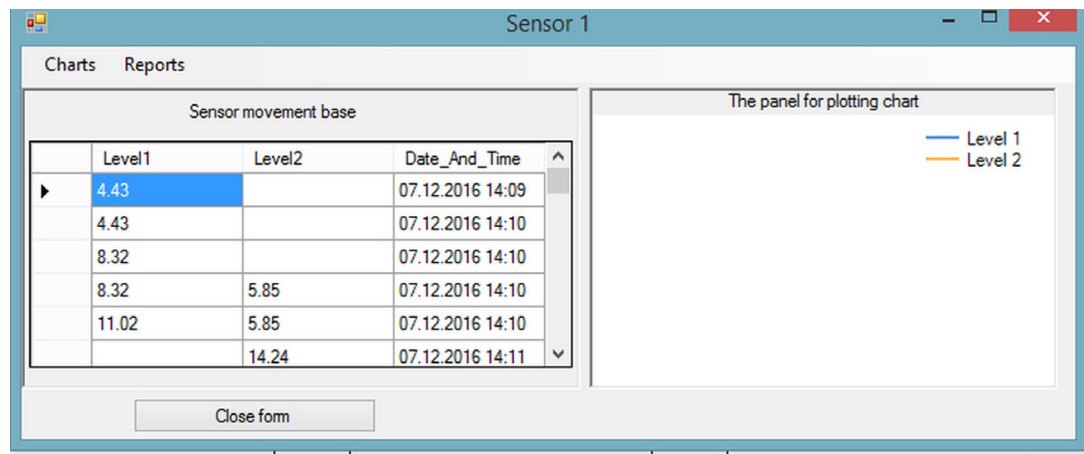

Fig. 3. Sensor 1 interface in the system for controlling the displacement of the mine workings.

In connection with the possible emergence of the need to work with the received data, including processing and analysis, it was decided to comprehensively export the received data to a Microsoft Office Excel document.

\section{Conclusions}

As a result of the work, the first version of the system for monitoring and recording the displacement readings in the mine workings roof was created, which will allow to record changes in the state of the roof of underground mine workings in real time. Based on the accumulated information, it is possible to predict the behavior of the roof in such mining and geological conditions, and also to analyze the causes preceding the change in the roof state (displacement, stratification, decompression). Monitoring the condition of mine workings will improve the safety of mining operations.

\section{References}

1. G. Molinda, C. Mark, El. J. Geotech Eng. 15, 547-588 (2010)

2. E. Kuzin, V. Shahmanov, D. Dubinkin, Web of conferences. 21, 03006 (2017)

3. B. Gerike, I. Panachev, E. Kuzin E3S Web of Conf. 15, 03008 (2017)

4. Z. Haijun, F. Ma, J. Xu, J. Guo, Int. J.of Rock Mech. \& Mining Sc. 53, 120 (2012)

5. M. Alvarez-Fernandez, C. Gonzalez-Nicieza, A. Menendez-Diaz, Eng. Geol. 80, 1 (2005)

6. A. Abramovich, E. Pudov, E. Kuzin, E3S Web of conf. 21, 01011 (2017)

7. V. Gogolin, Y. Lesin, A. Djagileva, E3S Web of Conf. 15, 01001 (2017)

8. A. Mufundirwa, Y. Fujii, J. Kodama, Int. J. Rock Mech. Min. Sc. 47, 1079 (2010)

9. Z. Chunbin, Z. Yubao, Z. Genu, L, Janji, M. Shuki, Sensors. 17, (2017)

10. J. P. Doherty, A. Hasan, G. Suazo, A. Fury, Canadian Geotechn. J. 52, 1901 (2015)

11. E Lawson, D. Tesarik, M. K. Larson, H. Abraham, 35th Int. Conf. on Ground Control in Mining. 27, 1 (2016)

12. Y. Zhao, N. Zhang, G. Si, Sensors. 16, 1759 (2016)

13. A. Catalano, F. Bruno, M. Pisco, A. Cutolo, A. Cusano, Sensors. 1418268 (2014)

14. Z. Z. Zhang, J. B. Bai, Y. Chen, S. Yan, Int. J. Rock Mech. Min. Sci. 80, 1 (2015)

15. B. Hebblewhite, J. Galvin, Int. J.of Rock Mech. \& Mining Sc. 27, 3 (2017) 